\title{
Beitrag zur Glaucom-Lehre.
}

\author{
Von
}

J. Jacobson sen. in Königsberg i. Pr.

Nicht weniger als fünfzehn Jahre hatte die Hypersecretions-Lehre ihre Kraft in verschiedenen Definitionen erschöpft, bis Graefe sein Unvermögen, den Krankheitsprocess zu erkennen, bescheiden eingestand, - und wiederum vergingen fünfzehn Jahre, bis die Retentions-Lehre, die bei den Ophthalmologen so viel Beifall gefunden hatfe, einen Stoss erhielt, von dessen tödlicher Wirkung sie nur noch für kurze Zeit durch ihre Elasticität und Liebe zum Leben bewahrt bleiben kann.

Birnbacher und Czermak haben in diesem Archiv den augenfälligen Beweis für venöse Stasen in dem vorderen Segmente der Chorioidea, wie sie nach Stellwag v. Carion in neuester Zeit von Arlt und mir hauptsächlich angenommen waren, geliefert, - die alte Chorioiditis ist wieder in ihr Recht eingesetzt, - eine secundäre Periphlebitis mit Verengung grosser Venen und besonders der Vasa vorticosa ist nachgewiesen, - eine zuverlässige Beobachtung von Verwachsung der Iris-Peripherie mit der Cornea durch blosse Apposition will sich noch immer nicht finden, während die entzündliche Verwachsung über- 
zeugend durch Sectionen constatirt ist, - kurz von der neuen Lehre bleibt als winziger Rest nur die paradoxe Behauptung: "Glaucom ist ein Symptom." Auch ihr ist im Kampfe gegen die Elemente der allgemeinen Pathologie eine Prognosis pessima zu stellen.

Dass Birnbacher und Czermak in ihrer unter dem bescheidenen Titel: „Beiträge zur pathologischen Anatomie und Pathogenese des Glaucoms" veröffentlichten, ausgezeichneten Abhandlung (Archiv Bd. 32) den alten Streit für immer entschieden haben, durfte nicht verschwiegen werden; denn so lange jeder Autor sich zu einer beliebigen Glaucom-Theorie für berechtigt hält, so lange haben wir eben so viele, subjective Pathologien. Hielte ich Schnabel's Theorie nicht für einflusslos, so würde ich die schlagende Widerlegung, die ihr zu Theil geworden ist, ebenfalls ausführlicher referirt haben. Unter den obwaltenden Umständen. genügt es, die Thatsache zu constatiren. Den Einwand, dass Birnbacher und Czermak sich nur auf die kleine Zahl ron neun Sectionen stützen, kann ich nicht gelten lassen; es ist unwiderleglich nachgewiesen, dass in neun untersuchten Augen diejenigen Veränderungen, die nach de Wecker dem Begriffe "Glaucom" widersprechen, vorhanden waren. Dieser Nachweis genügt. -

Zwei Irrlehren sind beseitigt, aber eine neue, den Erscheinungen nicht widersprechende Lehre, ist noch nicht gefunden. Wenn Birnbacher und Czermak auch wiederholentlich die Absicht, den glaucomatösen Process zu erklären, leugnen, so wird man ihnen das Verdienst, die Sache durch gründliche Kritik. wie durch genaue Beobachtung, originelle Versuche und nene Gedanken gefördert zu haben, schwerlich bestreiten und nichts natürlicher finden, als dass das complicirte Problem anf dem von ihnen eingehaltenen, streng wissensehaftlichen Wege nicht im ersten Anlaufe gelöst worden ist.

Was sie erreichen wollten, überschreitet die Grenzen, 
die der Leistungsfühigkeit des Klinikers gesteckt sind. Er kann schnell zum Ziele gelangen, aber er kann das Erworbene nicht sicher behaupten, so oft es auch den Anschein haben mag, als sei er berufen, durch Beobachtung und Speculation zu antecipiren, was die Wissenschaft nur auf weiten Umwegen erlangt. Um seiner höchsten Aufgabe, der therapeutischen, rationell zu genügen, muss er allerdings das Wesen der Krankheit, dem die Behandlung sich accomodiren soll, erkannt haben, aber wie oft seine Mühe vergeblich ist, weiss Jeder, der ex nocentious vorgefasste Meinungen aufgeben und neue Wege einschlagen gelernt hat. "Beobachtung am Lebenden" ist das Mittel, "Combinationen" sind die unsicheren Wege, auf die er angewiesen ist, bis der pathologisehe Anatom ihn mit einem neuen, werthrollen Mittel, den "Krankheitsproducten", unterstützt. Wie sich beide ergänzen, wie jeder von Beiden nur auf seinem Gebiete dem Ganzen nuttzen kann, soll mit Bezug auf eine beiläufige Bemerkung in der Abhandlung von Birnbacher und Czermak das folgende Beispiel aus der Glaucom-Lehre illustriren.

Der Kliniker sei durch den Verlauf der Symptome bewogen worden, die Disposition zum Glaucom in „venösen Stasen" zu vermuthen, für die Ursache dieser letzteren mit Rücksicht auf das Alter der Kranken "senile Gefässveränderungen" zu halten! Dann bleibt ihm zunächst die relative Seltenheit des Glaneoms. Er mus von Nenem zu seinen Beobachtungen zurückikehren und findet, wenn auch nicht ausnahmlos, so doch als Regel, dass seine Kranken an geringer Energie der Herzthätigkeit gelitten haben. Niedriger Arteriendruck steigert die venöse Stase, die Hypothese, so weit der Kliniker sie aufstellen darf, ist fertig und erwartet ihre Censur vom pathologischen Anatomen. - Den glücklichen Fall nun weiter angenommen, dass der pathologische Anatom bei einigen Sectionen die Bedingungen für venöse Stasen in groben Gefäss - Ver- 
änderungen findet und letztere für die directe oder wesentlichste Ursache des Glancoms halt, so ist er in Zukunft auf die Unterstützung des Klinikers angewiesen; denn wie die Herzthätigkeit gewesen, ob psychische Depression und dergleichen mitgewirkt haben mag, sagt ihm, wenn die Veränderungen der Gefässe gering sind oder fehlen, der Sectionsbefund nicht.

In der mehrfach citirten Abhandlung kommt es zu keiner scharfen Trennung des klinischen und pathologisch-anatomischen Gebietes, die Verfasser beherrschen beide. Deshalb ist mir die Bestätigung und Erweiterung, die meine Ansichten durch ihre Untersuchungen gefunden haben, von ganz besonderem Werthe. Vielleicht glüekt es mir in nicht geringerem Maasse mit zwei besonders wichtigen Theilen der Glaucom-Lehre, dem Verhalten des Glaskörpers und der Sehnerven-Excavation. Was auch in dieser Beziehung klinische Beobachtungen gelehrt haben, liegt ihrer streng wissenschaftlichen Methode so fern, dass ich hoffe, ihnen einiges Neue zu bringen.

Was man erworben hat und was zunächst anzustreben ist, darüber sich klar zu werden, dürfte in jedem Stadium wissenschaftlicher Thätigkeit eine unerlässliche Bedingung weiteren Fortschreitens sein. Es gereichte mir deshalb nicht zu geringer Befriedigung, meinen seit Jahren vergeblich ausgesprochenen Wunsch, eine Verständigung über diejenigen Krankheitsbilder, die man Glaucom nennen wolle, zu suchen, von Birnbacher und $\mathrm{Czermak}$ gebilligt zu finden. Auch in der Ophthalmologie setzt jede wissenschaftliche Discussion voraus, dass Alle mit den Worten, derer sie sich bedienen, denselben Sinn verbinden, während wir seit der Erweiterung des alten Krankheitsbegriffes durch Graefe über ein „Glaucom" sprechen und schreiben, dessen Grenzen jeder nach seinem subjectivem, den Anderen unbekanntem Ermessen bestimmt. Dass man in klarer Erkenntniss der Schwierig- 
Teiten zu meiner Forderung gesehwiegen hat, wundert mich nicht, aber dass man mir beweisen würde (!), wie zweckmässig es sei, Worte, unter denen man theoretisch nicht dasselbe verstehe, in der Praxis so zu brauchen, als ob man über ihren Sinn vollkommen einig sei, das überstieg allerdings meine Erwartungen.

Auch in einer zweiten, das Wesen des Glawcoms betreffenden Ansicht habe ich mich voller Uebereinstimmung zu erfreuen gehabt. In den Mittheilungen aus der Königsberger Universitäts-Klinik (1879) ist gezeigt, dass Graefe's "Drucksteigerung mit Rückwirkung anf Retina und Opticus" mit seiner Eintheilung der Glaucome in primäre und secundäre principiell unvereinbar sei: das Eintheilungsprincip ist nämlich nicht im Wesen des Glaucoms, sondern in unserer Fähigkeit, die intraoculären Ursachen des Glaucoms zu erkennen, enthalten. So unbedentend und theoretisch es erscheinen mag, für die Unglücklichen, die in ihrem ärztlichen und Lehrberufe immer noch dadurch gestört werden, dass sie nicht wissen, was als Glaucom anzusehen und zu behandeln ist, werden dergleichen theoretische Fragen eminent practisch.

Bedentungslos aber sind sie allerdings gegen die Entscheidung über die Natur des acuten Glancoms. Es ist weder die Lymphe, noch ihr verhinderter Abfluss, deren endliches Verschwinden bei der Auferstehung der Chorioiditis für mich besonderen Werth hat, sondern vor Allem, dass wir mit unserem Glaucom nicht aus den weiten Gebieten der. bisherigen pathologischen Vorstellungen heraustreten, dass wir eines der ernstesten Krankheitsbilder, das acute Glaucom, nicht davon abhängig machen, ob die Lymph-Emissarien etwas mehr oder weniger obstruirt sind, und dass wir, anstatt mit der uncontrollirbaren Lymphe, der man um so mehr pathologische Wunder andichten kann, je weniger man von ihr weiss, mit Blutflüssigkeit und Blutgefässen zu thun haben, deren physiologisches und pathologisches Verhalten 
bei aller Lückenhaftigkeit unseres Wissens soweit bekannt ist, dass wir nicht jede Hoffnung anf eine klarere Einsicht in den räthselhaften Process anfzugeben brauchen. Ausserdem aber bekenne ich gern - und ich müsste wenig Passion fur meinen klinischen Beruf haben, wenn es anders wäre, - dass es für die Mühe und Arbeit des Klinikers keinen schöneren Lohn giebt, als die Gewissheit, durch Beobachtung und Combination das Wesen der Krankheiten annähernd erkannt zu haben.

Wer das alte, inflammatorische Glaucom zu Grunde legen will, um zu wissen, wie weit er den Begriff ausdehnen darf, fur den ist die Niederlage der Lymph-Hypothese nicht etwas rein Theoretisches. Wir dürfen mit den venösen Stasen und den inflammatorischen Processen, deren Vorkommen nicht mehr bestritten werden kann, rechnen, wenn auch der empirische Beweis ihrer Constanz im Augenblick unmöglich ist, und können es den Gegnern überlassen, ihre Pathologie ohne empirisches Fundament weiter zu treiben. Unsere nächste Aufgabe ist durch das Krankheitsbild gegeben: von den constanten, charakteristischen Symptomen (der Drucksteigerung und der Rand-Excavation) haben wir auszugehen, um zu. sehen, in wie weit sie uns über das Wesen des Glaucoms Aufschluss geben.

In früheren Mittheilungen habe ich auf die Härte des enucleirten Auges aufmerksam gemacht und aus derselben auf Veränderungen im Inhalte des Glaskörperraumes geschlossen. Was uns die pathologische Anatomie vom glaucomatösen Glaskörper an zerstreuten Mittheilungen über Sectionsbefunde gebracht hat, ist zu dürftig und zusammenhanglos, als dass es sich für eine Pathologie des Glaskörpers schon verwerthen liessè. Klinische Beobachtung aber und der Verlauf der Iridectomie lehrt, dass sich kaum bei einer anderen Augenkrankheit so mannichfache Veränderungen nachweisen lassen, als bei den verschiedenen Formen des Glaucoms. 
Die Palpation eines normal gespannten Auges, wie wir sie zur Bestimmung des intraocularen Drucks mit zwei hinter dem convexen Knorpelrande auf die Lidhaut wirkenden Fingerspitzen auszuüben pflegen, giebt mir über gewisse Details keine sichere Auskunft, ich vermag den Inhalt des Bulbus gegen die Sclera nicht abzugrenzen, finde anch keinen Unterschied in der Resistenz zwischen den peripheren Partieen und der mittleren Substanz des Glaskörpers. Durch die Iridectomie wird hierin nichts geändert; collabirt das Auge nicht gar zu sehr, so ist die Spannung etwas vermindert, die Sclera scheint dem Inhalte anzuliegen, das ganze Auge etwas kleiner zu sein. Es gehört zu den seltenen Ausnahmen, dass ähnlich, wenn auch in viel geringerem Grade, wie bei mancher Phthisis bulbi mit Knorpelbildung im Corpus ciliare, die Sclera sich über einen in der Tiefe fühlbaren, harten Körper hin und her schieben lässt, als ob sie für den Inhalt zu weit geworden wäre.

Die Palpation des Glaucoma simplex mit enger Kammer und fühlbarer Drucksteigerung ergiebt vor der Iridectomie denselben Befund bei erhöhter Resistenz. $\mathrm{Nach}$ der Iridectomie unterscheide ich deutlich den festen, kugligen Glaskörper, über den sich die Selera verschieben lässt. Er erseheint nur kleiner, als vor der operation. Wie selten etwas Aehnliches, oder wie geringe Grade ausnahmsweise in nicht-glaucomatösen Augen vorkommen, weiss ich nicht, im glaucomatösen Auge kenne ich seit etwa fünf Jahren den angegebenen Befund als Regel ohne Ausnahme.

Ist die Beobachtung richtig, so ist entweder mit dem Humor aqueus resp. nach der Iridectomie Glaskörperflüssigkeit durch die Wunde ausgetreten, oder der Glaskörper hat Wasser auf vorläufig unbekannte Weise abgegeben. Bei enger, vorderer Kammer ist die Quantität des Humor aqueus so gering, dass einige Tropfen mehr 
oder weniger der Aufmerksamkeit kaum entgehen können, bei partieller Verflussigung pflegt das Auge weicher zu sein, auf geringen Fingerdruck Wasser durch die Wunde auszutreten. Nichts von Allem lässt sich nachweisen. Aus diesen Gründen glaube ich, dass der Glaskörper in unmittelbarem Ansehlusse an die Operation kleiner, fester und ärmer an Wasser wird.

Die Palpation bei acutem Glaucom ergiebt vor und nach der Iridectomie keinen Unterschied von Hülle und Inhalt, die Spannung lässt gewöhnlich nicht erheblich nach, um bald zu steigen. Entwickelt sich, wie es bei dem Glaucoma simplex noch häufiger vorzukommen scheint, nach der Iridectomie ein Glaucoma malignum, so nimmt die Spannung ohne wahrnehmbare Ausdehnung der Sclera täglich zu, bis zur Consistenz eines vollkommen festen, soliden Körpers, - eine hinter dem Aequator durch die Sclera 4-6 $\mathrm{mm}$ tief in das Innere gestossene Reclinations-Nadel oder ein Graefe'sches Messer entleert kaum einen Tropfen Flüssigkeit, - eine breite Nadel, uber das Centrum (also etwa 12-14 $\mathrm{mm}$ ) in das innere Ange eingedrungen und schnell zurückgezogen, lässt zwei bis drei Tropfen einer zăhflüssigen, dunkelgelben Substanz austreten, ohne dass das Auge fühlbar weicher wird oder auf Druck weiter reagirt. Der Glaskörper des Glaucoma malignum ist wasserarm, eine klebrige Substanz von dunkelgelber Farbe, anscheinend nicht grösser, als ein normaler.

Ueber die Beschaffenheit des Glaskörpers während eines Anfalles von Glaucoma acutum ist aus nahe liegenden Gründen durch Punctionen nicht viel ermittelt worden. Unter geübten Ophthalmoskopikern sind es vorzugsweise Schweigger und de Wecker, die mit zunehmender Energie, je länger der Streit dauert, die ganze, sogenannte Medien-Trübung in die Cornea verlegen, den Humor 
aqueus und das Corpus vitreum durchaus klar sein lassen. Vielleicht wird ihre apodiltische Behauptung etwas an Sicherheit verlieren, wenn sie von der Chorioiditis anterior der Collegen Birnbacher und $\mathrm{Cz}$ ermak Notiz genommen haben werden.

Meinen Erfahrangen nach nehmen an einer gewissen Intensität der Trübung immer sämmtliche Medien Theil. Für die Trübung des Humor aqueus habe ich mich in diesem Archiv bemüht, Beweise, die mir unwiderleglich scheinen, beizubringen, aber in Sachen der Beobachtung wird wenig Werth auf Beweise gelegt, so lange die Sinne das grosse Wort führen. Ich habe deshalb - für den vorliegenden $Z$ weck ausreichend - die Frage nicht mehr allgemein gestellt, sondern auf Ausnahmen eingeengt: „Können im Verlaufe des acuten, nicht complicirten Glaucoms heilbare, diffuse Glaskörpertrübungen vorkommen, oder bleibt der Glaskörper ausnahmslos klar?" Zur Beantwortung dieser Frage pflege ich meinen Zuhörern in jedem Semester einen Fall vorzustellen, der ungefähr in folgendes Schema hineinpasst: „Glaucoma acutum mit oder ohne Prodrome, bis zur Erkrankung scharfsichtig. Hohe Spannung - dichte, diffuse Medien-Trübung, - Sehvermögen auf quantitative Lichtperception reducirt. Iridectomie obne Hindernisse, ohne Blutung, Heilungsverlauf subjectiv normal. Erste Sehprobe nach etwa 14 Tagen bei scheinbar schwarzer Pupille, - Resultat: Bewegungen der Hand, allenfalls Zahl der Finger in grosser Nähe. Ophthalmoskopisch: matter, graurother Hintergrund-Reflex, keine Einzelheiten sichtbar, Linse und Cornea klar. Seitliche Beleuchtung: in der Hornhant kaum eine matte Andeutung der alten Trübung, linse nicht getrübt. - Zweite Sehprobe nach drei Wochen: $\mathrm{S}=\mathbf{1}$, alle Functionen normal. Die intraoculare Trübung ist allmählich heller geworden, schliesslich verschwunden, ohne sich in Flocken aufzulösen."

v. Graefe's Archiv für Ophthalmologie, XXXIV. 1. 
In der Regel hellen sich die Medien gleichzeitig auf, das skizzirte Schema gehört zu den Ausnahmen, genügt aber als Beweis für folgende Behauptung: es giebt eine mit dem acuten Glaucom-Anfalle auftretende und die Iridectomie einige Wochen überdauernde, diffuse Glaskörpertrübung.

Das Glaucoma congenitum (Buphthalmos) und das Glaucom jugendlicher Individuen mit progressiver Myopie zeigt in Bezug auf die Beschaffenheit des Glaskörpers gleiches Verhalten. Das letztere habe ich in zwei Formen beobachtet, von denen die eine sich durch periodische, migräneartige Anfälle mit starker Verdunklung (Medien-Trübung) charalterisirt, die zweite unter dem Gefühle von unangenehmer Spannung des Auges, die sich beim Arbeiten steigert, gleichmässig verläuft. Der Augapfel ist gleichmässig stark ausgedehnt, dadurch bervortretend, die ganze, sichtbare Sclera bläulich, vordere Kammer sehr tief, Pupille etwa mittelweit, reagirend, Papilla optica tief excavirt, Spannung hoch, Sehschärfe sehr gering.

An der ersten Form habe ich bei der Iridectomie nichts Auffalliges bemerkt, für die zweite und für diejenigen Falle von Buphthalmos, die ohne starke Blutungen verlaufen, gilt Folgendes: Nach der Incision mit der Lanze in der Corneo-Seleral-Grenze entleert sich sehr viel mehr Flüssigkeit, als die vordere Kammer trotz ihrer Tiefe enthalten kann. Beendet man, um die Iris nieht zu tief zurücksinken zu lassen, die Operation 'so schnell, als möglich, so wiederholt sich der Ausfluss klarer Flüssigkeit, die allmählich ein wenig klebrig wird, der Augapfel collabirt, wie nach Punetionen der Amotio retinae, und trotzdem tritt immer neues Wasser aus, sobald die Wundränder durch leisen Druck zum Klaffen gebracht werden. Die Palpation lässt auch bei tiefstem Druck nichts Resistentes im Innern fühlen. - Bisher habe ich 
nicht nur ausnahmslos gute Heilungen beobachtet, sondern ausserdem eine erhebliche Reduction der Ausdehnung, Aufhören der Schmerzen, keine Recidive, aber auch keine Besserung des Sehvermögens (längste Beobachtungszeit: 7 Jahre). - Kommt es bei schnell fortschreitender Myopie jugendicher Individuen zu Glaucoma simplex, so vertritt die Stelle des normalen Glaskorpers eine in Farbe und Consistenz dem Humor aqueus gleiche Flüssigkeit, durch deren Abundanz die Lamina cribrosa tief excavirt und die Sclerotica nach allen Richtungen erheblich gedehnt wird. Da der Process durch Iridectomie coupirt werden kann, bleibt die Frage, ob die Secretion vermindert oder der Abfluss erleichtert wird, vorläufig offen.

Was bisher über die Beschaffenheit des glaucomatösen Glaskörpers gesagt worden ist, fasse ich kurz zusammen: Von der totalen Verflüssigung der jugendlichen Myopen, die nach der Iridectomie keine Resistenz des Inhaltes fühlen lässt, kommen wir zum Glaucoma simplex, dessen Glaskörper während der Iridectomie Wasser verliert und bei nicht ganz oberflächlicher Palpation als eine etwas kleinere, feste Kugel, die das Auge nicht vollkommen auszufüllen scheint, gefühlt wird. Der Glaskörper des Glaucoma acutum giebt nach der Iridectomie, wie es nach der Palpation scheint, kein Wasser ab, - er kann, wie die diffuse Trübung zeigt, weniger transparent, er muss, wie die Palpation vor der Operation lehrt, resistenter sein. Das Glaucoma malignum lässt eine erhebliche Vergrösserung nicht annehmen, aber vermehrte Festigkeit, Umwandlung in eine zähe, gelbliche, aus Einschnitten kaum einige Tropfen Flüssigkeit entleerende Substanz ist sicher nachgewiesen.

Wie es zugeht, dass die Lamina cribrosa dem Drucke einer intraocularen Flüssigkeit, deren Quantität den Augapfel gleichmässig um $4 \mathrm{~mm}$ Achsenlänge und mehr aus- 
dehnt, allmählich nachgiebt, bedarf keiner weiteren Erklärung. Ist aber, wie es nach der Härte enucleirter Augen den Anschein hat, die Drucksteigerung nicht ron der Höhe des Secretions-Druckes, sondern von dem Iruhalte des Glaskörperraumes, der gewöhnlich einen normalen Glaskörper an Umfang nicht übertrifft, abhängig, so ist das Zustandekommen der. Excavation weniger selbstverständlich. Um dasselbe zu verstehen, habe ich, was eigene Erfabrungen und Mittheilungen Anderer mich uber die pathologischen Beziehungen zwischen Corpus vitreum und Papilla optica im Allgemeinen gelehrt haben, einer Revision unterworfen. Das Resultat, so weit mir dasselbe für meinen Zweck verwerthbar zu sein scheint, bringt der folgende Abschnitt.

Von den nur zu zahlreichen Atrophien der Papilla optica, von der Mehrzahl der entzündlichen Schwellungen, von der Embolie der Central-Arterie wissen wir, dass sie aus dem acuten Stadium in ein langes, chronisches übergehen können, ohne den Glaskörper merklich zu rerändern. Von Blutungen aus grossen Gefässen der Papille, der Retina und von den, wie mir scheint, nicht selten aus der Macula lutea in den Glaskörper gelangenden, grossen Haemorrhagien, wie von Manz's Retinitis proliferans, die ich ebenfalls zu den Haemorrhagien des Opticus uud der Retina zähle, dürfen wir annehmen, dass sie sich nicht auf praeformirten Wegen verbreiten. Die Höhe des Blutdruckes, unter dem die GefässRuptur erfolgt, die Masse des Extravasates, der Widerstand der nächsten Umgebung dürten eher Factoren sein, von denen die Richtung, welche die Blutmasse einschlägt, vorzugsweise abhängt.

Diesen mehr weniger häufigen Spiegelbildern stehen seltenere Fälle gegenüber, deren typisches Auftreten eine anatomische Praedisposition, einen bestimmten, angewiesenen Verbindungsweg zwischen Papille und Glaskörper vermuthen 
lässt. Wie mich die neueste Literatur der durch Samelsohn, Vossius, Nettleship u. A. anatomisch erklärten Intoxications-Amblyopien und retrobulbären Entzündungen annehmen lässt, ist es wenig bekannt, dass man mitunter bei unzweifelhafter Tabaks-Amblyopie aus dem CentralCanale der Papille eine formlose, graue Masse auftauchen sieht, welche entweder - genau entsprechend der von Magnus in Zehender's Monatsblättern mitgetheilten und abgebildeten Vernarbung einer Ruptur der Retina und Chorioidea am gelben Flecke - über die temporale Hälfte der Papille zur Macula, oder in der Richtung des Cloquetschen Canals in den Glaskörper hineinwächst, in beiden Fällen, sobald sie die Papille verlässt, als bandartige, grellweisse, stark reflectirende Substanz lange stationär bleibend, bis ihre sehr lange Rückbildung beginnt.

Bekannter dürfte es sein, dass im Laufe der Febris recurrens gleichzeitig oder abwechselnd mit kleinen, grauen Infiltraten der Cornea, mit Hypopien, mit grauen Auflagerungen auf der vorderen und kleinen, später zur Cataracta polaris posterior confluirenden Trübungen der hinteren Kapsel und mit axialen Opacitäten hinter der tellerförmigen Grube aus dem CentralCanal der Papilla optica ein grauer Zapfen nach vorn in den Cloquet'schen Canal hineinwäehst, in dem er, sich nach vorn zuspitzend, eine Länge von $10-12 \mathrm{~mm}$ erreicht, mit kleiner Amphitude hin und her schwingt, ehe er sich spurlos zurückbildet.

Aehnliches habe ich bei der Retinitis syphilitica simplex beobachtet und mitunter nach langer Zeit, an einem durchscheinenden, graulichen Plaque im Central-Canal, als letztem Residuum, wieder erkannt, wie ich ihn vor ca. 30 Jahren in den „Verhandlungen des Königsberger Vereins für wissenschaftliche Heilkunde" bei Gelegenheit meiner ersten Mittheilungen über Retinitis syphilitica besehrieben habe.

Casuistische Mittheilungen in der Literatur der Hintergrunds-Krankheiten haben nur wenig Neues gebracht, aber 
vor einer Reihe von Jahren schon hätten die Pathologen auf Stilling's einfache Methode, den Glaskörper-Canal hervortreten zu machen, mehr Werth legen sollen, lange ehe er auf der vorletzten, Heidelberger Versammlung in seinem Vortrage über "Glancom" selbst auf seine alten Untersuchungen zurückkam. Seit dieser Zeit hat uns nun Flemming in seinem schematischen Augen-Durchschnitte eine Abbildung des Cloquet'schen Canales gegeben, bei deren Anblick der ärgste Septiker kaum an einer freien Communication zwischen dem Centralkanale der Papille und der aus den Gefässen des vorderen Aderhaut-Segmentes zur tellerförmigen Grube gelangenden Flüssigkeit durch den Cloquet'schen Canal zweifeln dürfte. Die Papilla optica als Basis des kegelförmigen Canals anzusehen, hatte ich mich schon durch Merckel's und Schwalbe's anatomische Angaben gewöhnt und darauf hin die Entwickelung der Excavation ophthalmoskopisch beobachtet, aber die ganze Form, die Breite und namentlich das obere Stück des Canals, wie Elemming es abgebildet hat, scheinen mir den Gedanken an eine Verbindung zwischen dem Corpus ciliare und dem Centralcanal, bis ein Irrthnm nachgewiesen wird, geradezu herauszufordern.

Stelle ich die zeitlich mit Beseitigung der venösen Stase zusammenfallende Wasserabgabe des Corpus vitreum bei Glaucoma simplex, seine Verdichtung bei entzündlichem Glaucom auf der einen, die pathologischen Immigrationen aus dem Corpus ciliare und der Papille mit den anatomischen Verhältnissen des Cloquet'schen Canals auf der anderen Seite zusammen, so komme ich zu folgendem Schlusse: Der (abgesehen von Herzthätigkeit, Athmung etc.) mit der Pupillenberegung und Accommodation fortwahrend wechselnde Blutdruck in den Ciliarfortsätzen und Venen bis zur Ora serrata bedingt eine ebenso 
wechselnde Filtration in den Glaskörpor. Der Cloquet'sche Canal mit seiner Einmündung in den Centralcanal der Papille ist das SicherheitsVentil zum Schutze des Sehnerven gegen einen mit chorioidalem Filtrat überladenen Glaskörper.

$\mathrm{Ob}$ die pathologische Anatomie im Stande sein wird, diese Annahme abzuweisen oder zu bestätigen, lasse ich dahingestellt sein. Jedenfalls dürfte eine Anzahl mikroskopischer Schnitte aus nicht zu späten Stadien der Excavation nach Glaucoma acutum, wenn sie gleichzeitig den Centralcanal der Papille und den Cloquet'sehen Canal treffen, für unsere Auffassung des Glaucoms und mancher anderer Krankheiten brauchbar sein. Den Leser bitte ich, mit der aus verschiedenen Beobachtungen zusammengestellten Hypothese nicht zu streng in's Gericht zu gehen, so lange dieselbe den Krankheits-Erscheinungen nicht widerspricht. Wie wir uns auch die Lösung unseres Problems denken mögen, immer werden wir, wie mir scheint, früher oder später an einen Punkt kommen, über den wir uns ohne Hypothesen nicht forthelfen können, nämlich über die Gesetze, unter denen in pathologisehen Fällen der Austausch $z$ wischen Blut- und Gewebs-Flüssigkeit zu Stande kommt.

Dass der Augenspiegel direct nicht viel über die Beziehungen des glaucomatösen Glaskörpers zur Papilla optica aussagen würde, war zu erwarten; denn während die trüben Medien der inflammatorischen Fälle eine genaue Untersuchung des Hintergrundes unmöglich machen, lässt die gleichmässige Transparenz des Glaskorpers im Glaucoma simplex nicht unterseheiden, ob der unsichtbare Cloquet'sche Canal von einer optisch dem Corpus vitreum identischen Flüssigkeit durchströmt wird.

Auch ron den Veränderungen der Papilla optica nahm ich mit Recht an, dass es selbst der beharrlichsten Beob- 
achtung nicht gelingen werde, der Entwickelung der Formveränderung durch eine unendliche Reihe kleinster Modificationen und Uebergänge zu folgen. Aus gröberen, constanten Eigenschaften der Excavation aber, aus ihrem Verhältniss zu den functionellen Störungen, vielleicht sogar aus Abweichungen ron der Regel (exceptionellen Formen des Sebnervenleidens) eine richtige Vorstellung der Vorgănge während des Lebens zu gewinnen, schien mir an sich nicht ausgeschlossen.

Was ich auf diese Weise für den vorliegenden Zweck festgestellt habe, soll in Kürze zusammengefasst werden, nachdem es in meinen früheren Beiträgen eingehender besprochen worden ist: 1 . Jede glaucomatöse Excavation geht vom Central-Canale aus, an einem Theile seiner Peripherie wird die Gefässknickung, in seiner Tiefe die Lamina cribrosa zuerst sichtbar; 2. erreicht dieselbe in der oberen oder unteren Hälfte den Rand, wâhrend die entgegengesetzte Hälfte in Niveau bleibt, so entspricht der Gesichtsfeld-Defect der Excavation, also Fehlen des unteren Gesichtsfeldes bei Excavation der oberen, des oberen bei Excavation der unteren Papillenhalfte; 3 , eine RandExcavation, bei welcher die Substanz der Papille im normalen Niveau bleibt, ist noch nicht beobachtet worden, die Rand-Excavation sollte mithin nicht im Gegensatze zu weniger peripheren Excavationen als charakteristisch für Glaucom angesehen werden, sondern jede vóm Centralcanale aus centrifugal fortschreitende Excavation; der Vorschlag ist praktisch wichtig, denn erst in einem späten Stadium, in dem die Iridectomie wenig leistet, pflegen die Gefasse am Rande der Papille zu verschwinden; 4. aus der Gefässverschiebung am Rande darf nicht, wie Graefe es that, auf eine Dehnung oder Knickung der Sehnervenfasern geschlossen werden. Es genügen wenige, genaue Gesichtsfeldmessungen, um sich von der Riehtigkeit dieses Satzes zu überzeugen, den, wenn 
ich nicht irre, zuerst Mauthner mit guten Gründen offentlich vertheidigt hat.

Die Zahl der Beobachtungen, die ich für unbedingt beweisend halte, ist nicht gross. Rand-Excavationen sind zwar häufig genug, und auch an solchen fehlt es nicht, in denen die Austrittsstelle der Central-Gefässe am weitesten nach rückwärts dislocirt ist, aber Exemplare von rechtwinkliger Gefässknickung an der Peripherie des Canals in ausreichender Menge zu sammeln und so lange, bis die Retina-Gefässe am Rande der Papille gleichsam coupirt sind, unter Augen zu behalten, fordert viel Zeit und günstige Gelegenheit. Unter meinen Beobachtungen ist keine, in der nicht die von der Peripherie des Central-Canals beginnende, diagonal oder horizontal nach der Schläfenseite ausstrahlende Excavation ohne ortliche Unterbrechung vorgeschritten wäre, d. h. längerer Stillstand in einer zwischen Mitte und Rand liegenden Zone kam oft genug zar Beobachtung, aber nie eine zwischen zwei Vertiefungen in normalem Niveau gebliebene, höher gelegene Partie, - ferner schritt die Einsenkung nie längs der Peripherie fort, ehe das zwischen der Austrittsstelle und dem Rande liegende Gefässstück vorangegangen war, - dann blieb in der Regel, wenn die Lamina cribrosa schon dem Centralcanal entsprechend und in der ganzen, temporalen Papillenhälfte deutlich sichtbar war, in der nasalen Hälfte der Uebertritt der Retina-Gefässe über den Rand normal, die Lamina cribrosa unsichtbar, weil von einem mächtigen, gerötheten Nervenfaser-Wall bedeckt, - und endlich pflegten die abwärts verlaufenden Gefässe früher, als die oberen, dislocirt zu erscheinen resp. $\mathrm{zu}$ verschwinden.

Dass in dem schliesslichen Bilde der totalen Excavation mit Schwund der Achsencylinder die ectatische Lamina cibrosa später das Wesentliche ist, hatte schon Heinrich Müller klar gemacht, später hatte man eingesehen, dass die erste Folge der Drucksteigerung nicht die Knickung der Nerven- 
fasern am Rande der Papille, sondern die Ausbuchtung der Lamina cribrosa sein müsse. Von ibrem ersten Zurücktreten bis zum Schlusse haben Birnbacher und Czermak mit der ihrer ganzen Abhandlung eigenthümlichen Genauigkeit die physicalischen, nothwendigen Consequenzen in ihrer Formveränderung theoretisch demonstrirt und experimentell darzustellen versucht; aber je vollkommener das physicalisehe Gesetz zur Erscheinung kommen soll, desto constanter und einfacher müssen die Bedingungen sein, unter denen man seine Wirkungen za beobachten beabsichtigt. Hätten uns nicht genug Sectionen gelehrt, dass in tiefen OpticusGruben Glaskörperreste, Fetzen der Hyaloidea, entzündliche Producte, neu gebildete Gefässe u. dgl. m. vorkommen, so würde sich die Frage, auf welche Weise der Zwischenraum zwischen dem Glaskörper and dem Boden der Grube ausgefüllt werde, von selbst aufgeworfen haben.

In alteren Mittheilungen war ich von der Voraussetzung, dass das lockere Zellgewebe im Centralcanale der Papille zuerst zurückweiche, der Glaskörper nachrücke und von hier aus im interfascikulären Bindegewebe resp. den Achsencylindern selbstständige Reizungszustände erzeuge, kurz, dass die Excavation als ein Product zweier Factoren, der Drucksteigerung und unbekannter, von dem nachrückenden Glaskörper inducirter Ernährungsstörungen zu betrachten sei, ausgegangen.

Nach der hentigen Glaskörper-Anatomie wüsste ich in dem Gedankengange Niehts zu ändern, würde aber den ,nachrückenden Glaskörper" dureh eine vom Cloquet'schen Canale continuirlich in den Centralcanal sich entleerende Flüssigkeit ersetzen, durch eine aus vorderen Chorioidealvenen in den Glaskörper filtrirende Flässigkeit, deren Quantität von dem Grade der venösen Stase, deren Qualität von einem mehr hydropischen oder mehr entzündlichen Process (der von Birnbacher und Czermak beschriebenen Chorioiditis) abhängig ist. 
Ehe ich weiter gehe, will ich die Consequenzen des soeben Erörterten resummiren. Bekanntlich liess Graefe sich in seiner ersten, klassischen Abhandlung über Glaneom (Archiv Bd. III) durch sein Bestreben, die intraoculäre Drucksteigerung allein für den ganzen Krankheitsprocess verantwortlich za machen, wie er sehr bald selbst gestand, etwas zu weit hinreissen. Aus der Moglichkeit, jedes Symptom als Consequenz intraoculärer Drucksteigerung aufzufassen, wurde bald eine Wirklichkeit, die den ganzen Symptom-Complex und jedes Symptom für sich allein durch Drucksteigerung erzeugte, aber es währte nicht lange, bis an der Anaesthesia corneae, der Iridoplegie, dem Aussehen der Iris und allen objectiv wahrnehmbaren Anomalien die pathologischen Gewebsveränderungen ihren Antheil für sich reclamirten.

Mit meinen Erklärungsversuchen würde auch das letzte, reine Druck-Symptom, die Excavation, ihre r ein physikalische Ursache verlieren, wir würden mit Berücksichtigung der prodromalen und subacuten Anfälle den inflammatorischen Glaucom-Process folgendermassen aufzufassen haben: bis zu einer gewissen Grenze werden Stasen im vorderen Abschnitte der Choriodoidea durch Abfluss der filtrirten Flüssigkeit nach dem Centralcanale der Papilla optica, durch Erweiterung collateraler Gefässe, durch Resorption ödematëser, selbst entzündlicher Fluida ausgeglichen. Ueber diese Grenze hinaus kommt es zu stationären Gewebsveränderungen, die bei erhöhtem intraocularem Drucke ein anderes Krankheitsbild, als gleichartige Processe, in normal gespannten Augen darbieten.

"Drucksteigerung mit consecutiver Functionsstorrung des N. opticus und der Retina" ist, wenn wir noch andere Ursachen der Amblyopie zulassen, nicht mehr, wie Graefe annahm, das Wesen des Glaucoms. "Glaucomatöse Drucksteigerung" ist 
die bald stationäre, bald transitorische Consequenz einer hydropischen ödematôsen oder entzündlichen Schwellung des Glaskörpers auf dem Boden venöser Stasen der Chorioidea.

Ueber die ersten, nicht vom Drucke abhängigen Veränderungen der Papilla optica wird uns die pathologische Anatomie lange noch einen genügenden Aufschluss nicht geben, über die letzten besitzen wir eine nicht mehr kleine Zahl kurzer Andeutungen, die der Ordnung und Vervollständigung bedürfen, von dem Verhältnisse zwischen dem Inbalte der Excaration und dem Cloquet'schen Canale weiss man, wenn ich nicht irre, noch Nichts, Anch was ich mit dem Augenspiegel in Fällen von Glaucoma simplex beobachtet habe, ist lange nicht zum Abschlusse reif. Erwähnen, oder vielmehr mit aller Reserve andeuten, möchte ich, dass sowohl sehr tiefe Excavationen eines engen Centralcanales, als auch nicht zu späte Stadien des Glaucoma simplex mit Verdrängung der Gefässpforte nach der nasalen Hälfte in den excavirten Theilen mir eine ungewöhnliche Helligkeit und Lichtreflexe gezeigt haben, die ich nicht anders als aus einer wasserhellen, in die Papille eingedrungenen Flüssigkeit zu deuten im Stande war. Die Erscheinung überrascht am meisten, wenn man sich mit einem der bekannten Refractions-Spiegel für den hinteren Abschnitt des Glaskörpers einstellt und dann allmählich dem Auge nähert. Ist der Beobachter der Excavation nicht stark hypermetropisch, so ist der Unterschied zwischen der Licht-Intensität des weisslichen, von nicht excavirten Partien der Papille reflectirten Scheines und dem spiegelnden Glanze der benachbarten Excavation ausserordentlich auffallend. Bestätigte sich die Richtigkeit der Beobachtung, so läge ein Zusammenhang zwischen dem Wasserverluste des Glaskörpers nach der Iridectomie bei Glaucoma simplex und dem Abfluss durch den Cloquet'schen Canal nach der Papilla optica nicht all' zu fern. 
Unreife Beobachtungen den Lesern zur Begutachtung anzubieten, liegt weiter nicht in meiner Absicht, aber ob sich allgemein bekannte, bisher mit der Druck-Hypothese unvereinbare Symptome ohne Zwang aus der Annahme einer continuirlichen Communication der vorderen Chorioidea mit der Papille erklären lassen, darüber wenige Bemerkungen hinzuzufügen, dürfte man mir nicht verwehren.

Nach anamnestischen Angaben der Kranken kann ich annehmen, dass einige Wochen vergehen, ehe dem acuten Anfalle die Excavation folgt. Selbst Erfahrungen darüber zu sammeln, haben wir keine Gelegenheit; wir dürfen nicht experimenti causa Excavationen entstehen lassen, wenn wir Glaucom heilen sollen.

Wie viel Zeit vergeht, bis es nach einem acuten Glaucom-Anfalle zur Rand-Excavation kommt, können wir heutzutage nicht mehr bestimmen. Wir sollen Glaucom heilen, dürfen es natürlich zur Excavation nicht kommen lassen, müssen uns mit der Thatsache, dass auf der Höhe des Anfalles Gefässknickungen nicht beobachtet sind, und mit älteren Angaben Graefe's, der eine Anzahl Wochen vergehen lässt, ehe die Rand-Excavation sich bildet. zufrieden geben. Nachträglich während des Ueberganges des Glaucoma acutum in ein entzündliches Glancoma chronicum bei nachlassendem, aber constant gegen die Norm gesteigertem Drucke entstehende Formveränderungen der Papille sind ausnahmslos Excavationen. Da ihre ersten Symptome den intensirsten, entzündlichen spät nachfolgen, so spricht die Wahrscheinlichkeit dafür, dass sie nicht in Folge von Entzündung, sondern durch Druck entstehen.

Es giebt aber ansnahmsweise auch Formveränderungen der Papille, die in den ersten Tagen des Anfalles entstehen, unter dem Namen der "entzünd- 
lichen Atrophie" beschrieben sind, nicht als Excavationen enden. Um ein Schema zu geben:

,Glaucoma acutum mit intensiver Medien-Trübung, 14 Tage lang vernachlässigt. Sehschärfe der Trübung entsprechend. Normale Iridectomie, Nachlass der subjectiven Symptome, Aufhellung der Medien, nach 14 Tagen erste Sehprüfung $S=1 / 10$, Se: stark eingeschränkt.

Ophthalmoskopisch: Medien klar, Grenzen der Papille durch weisse, in die Retina ausstrahlende Trubung verdeckt, weder Scleral- noch ChorioidalRing sichtbar, - Niveau das der Retina, Farbe der ganzen Oberfläche gleichmässig weiss, so dass auch der Centralcanal fehlt, Arterien und Venen eng, in der Retina etwas weiter, kleine Gefässe fehlen, Transparenz der Papille aufgehoben."

Fine Heilung habe ich nicht gesehen, ebenso selten erhebliche Verschlimmerung, wenn der Process nicht ganz friseh war. Die meisten Patienten, bei denen ich das Bild nach der Iridectomie gesehen habe, wussten von ihrer „Entzündung" seit 1 bis 2 Wochen Etwas. Ein Mal glaube ich den ersten Anfang beobachtet zu haben:

„Der vor Kurzem von einem Podagra-Anfalle genesene Kranke holte Nachmittags 4 Uhr meinen Rath, ob er in einer Stunde eine Geschaftsreise nach Berlin wagen dürfe, - seine Augen seien seit 12 Uhr etwas trübe und geröthet, aber kaum schmerzhaft. Es war der Anfang des ersten GlaucomAnfalles mit geringer Medientrübung, Pupillenerweiterung, Arterienpuls T. +1 .

Da die Familienverhältnisse eine Operation nur im dringendsten Nothfalle zuliessen, versuchte ich's mit Eserin. Nach 24 Stunden leichtes Oedem am Lidrande, Chemosis, vermehrte Injection, Mydriasis etwas stärker, - die Trübung gestattete nicht mehr 
ein klares Hintergrundbild zu erkennen, aber auffallend war ein weisser Reflex der Tags zuvor noch rothen Pupille und unmittelbar vor derselben ein continuirlicher, bei den Bewegungen des Auges unbeweglicher Schleier.

In den nächsten 24 Standen übernahm ich selbst, abwechselnd mit einer zurerlässigen Pflegerin, die Application des Eserin, das schliesslich Myosis und Entspannung des Auges bewirkte. Den Process, wie durch Iridectomie, durch Eserin zu coupiren, gelang nicht, er verschleppte sich durch 4 Wochen, während deren von Neuem energisch zu den Myoticis gegriffen werden musste, wenn ihre Wirkung unerwartet schnell nachliess, aber die Heilung kam ohne Tridectomie zu Stande.

Bei der Entlassung des Patienten waren beide Papillen scharf contourirt, die Grenzen gegen die Retina genau, dio des Centralcanals kaum sichtbar, Niveau normal, Transparenz nicht aufgehoben, Gefässe auf der Papille durch Verdicknng der Adventitia scheinbar blutarm, in der Retina normal, Glaskörper normal, Functionen normal. - Seit drei Jahren hahe ich den Fall in Beobachtung und notire in mein Journal: status idem. Den günstigen Verlauf schreibe ich der frühen Behandlung zu.

Ohne sein Wissen, aber hoffentlich nicht gegen seinen Willen, will ich noch kurz anführen, dass mein alter Freund Klebs (Zürich), dessen Beistand meinen ersten klinischen Stadien, wie Virchow's Archiv schon vor etwa 25 Jahren zeigt, nicht fehlte, auch in den letzten Jahren wieder mit der Glaucom - Frage pathologisch - anatomisch und experimentell-pathologisch sich beschäftigt und sofort für die venöse Stase gegen die Lymph-Hypothese entschieden hat. Wie er mir vor wenigen Wochen mündlich 
mittheilte, hat Einer seiner Schüler nach neuer Methode die von mir postulirte Communication in glancomatosen Augen nachgewiesen. Die Flüchtigkeit der gelegentlichen Mittheilung entzieht ihr leider einen Theil des grossen Werthes, den sie für mich haben würde.

Die sogenannten Opticus-A trophien nach Glancoma acutum, die ich soeben besprochen habe, sind nach der Zeit des Entstehens, nach ihrem Aussehen etc., unzweifelhaft entzündlichen Ursprungs, die eine folgende Krankheitsgeschichte lässt die entzündliche Verfärbung gleichzeitig mit einer Glaskörpertrübung vor dem Sehnerven auftreten, leider ist sie die einzige dieser Art. Mit der DruckHypothese waren diese Atrophien nicht vereinbar. Eben so wenig mit ihr vereinbar waren bekanntlich die seltenen Fälle von Glaucoma fulminans, die Graefe in diesem Archiv beschrieben, dieser und jener beobachtet hat: acute vollständig oder fast vollständige Frblindung unter starker Medientrübung bei mässigem Druck und geringer Excavation!

Das Glaucoma simplex (wassriges Filtrat) kommt weder als Glaucoma fulminans vor, noch giebt es nach der Iridectomie eine entzündliche Infiltration der Papille. Letztere sowohl, als auch die Erblindung des Glaucoma fulminans bei mässiger Excavation ist für diejenigen, welche eine Communication zwischen der vorderen Chorioidea und der Papille dureh den Cloquet'schen Canal annehmen, leicht verständlich.

Die klinischen und anatomischen Gründe, die mich bestimmt haben, eine Verbindung zwischen dem vorderen Theile der Chorioidea durch den Glaskörper-Canal nach der Papille hin anzunehmen, sind hiermit angeführt. Dass sie sich in der Sache selbst den Stilling'schen Untersuchungen anschliessen, macht sie mir um so plausibler. Ohne neue Data weiter zu gehen und Hypothesen über 
Stromesrichtung, über die Abschlusswege nach räckwärts, über Verengung oder Verschluss ron Emissarien, die man nie gesehen hat, zu machen, halte ich von unserem jetzigen, klinischen Standpunkte aus für unzulässig. Die Fontanaschen Räume können für lange Zeit als Warnung dienen. Wem sie nicht genügen, der lese die Litteratur der Glaskörper-Injectionen, damit er sich die Sache nicht allzu leicht vorstelle!

Wenn es dem Kliniker verwehrt ist, eine anatomische Norm von allgemeinem Charakter, wie z. B. das Vorhandensein einer Communication, weiter im Detail auszubauen und auf dieses Gemisch von Wahrheit und Dichtung eine neue Pathologie zu begründen, - so steht es ihm nicht nur frei, sondern ist sogar seine Aufgabe, die anatomische Voraussetzung auf ihre Verträglichkeit mit den ihm bekannten, pathologisehen Erseheinungen zu prüfen, wie es in Folgendem geschehen wird.

Klinische Erfahrung lehrt:

1. es giebt Glaucome mit Drucksteigerung ohne Rand-Excavation;

2. es giebt Rand - Excavationen ohne Drucksteigerung.

Das Glaueoma prodromale, subacutum und acutum, letzteres ehe die Acme erreicht ist, zeichnen sich constant durch Drucksteigerung aus. Die Papilla optica ist nicht excavirt, weil die Daner der Drucksteigerung und meist auch ihre Höhe nicht ausreicht, der Papille eine andere Form zu geben. - Die später während des Glaucoma chronicum inflammatorium nie fehlende Excavation ist vermuthlich in den meisten, wenn nicht in allen Fällen eine Folge der Drucksteigerung (Ectasie der Lamina cribrosa). Sicheres können wir nur durch Sectionen früher Stadien erfahren; denn eine Augenv. Graefe's Archiv für Ophthalmologie, XXXIV. 1. 
spiegel-Untersuchung verhindert die Medien-Trübung. Die Atrophia optica nach Glancoma acutum entsteht meist früher, als sie gesehen wird. Sie und das Glaucoma fulminans sind nach der Druck-Hypothese nicht zu verstehen, wohl aber, wenn man annimmt, dass pathologische Producte, die ans den Venen der vorderen Chorioidea herstammen, in die Papille eingeschwemmt werden kömnen. Die an der Papilla optica sichtbaren Symptome des Glaucoma acutum sind dureh die DrackHypothese allein nicht zu erklären.

Das Glaucoma simplex congenitum oder jugendlicher Myopen zeichnet sich durch hochgradige Drucksteigerung und meist tiefe Excavation aus. Das Verhalten der ungleichmässig gedehnten Sclerotica spricht dafür, dass auch die Exeavation eine Druck-Exeavation ist; denn es ist nicht denk bar, dass die Lamina cribrosa Widerstand leistet, wenn die Sclera nachgiebt. 'Die sehr viel häufigere Form des Glaucoma simplex im späteren Alter kommt bei erhöhtem, seltener normalem oder gar subnormalem Druck. zu Stande. Die ophthalmoskopische Untersuchung des Sehnerven in Verbindung mit dem Studium der Eunctionen, namentlich des Gesichtsfeldes, lehrt, dass die Excavation rom Centralcanale der Papille ausgeht und sich dann bald in einer, bald in der anderen Richtung verbreitet, dass das ophthalmoskopische Bild der Gefässknickung am Rande sich mit einer Knickung der Nervenfaser nicht deckt, und dem Orte des Gesichtsfeld-Defectes nicht entspricht.

Dass die Excavation bei subnormaler Spannung nur durch die willkürliche Annahme einer noch excessiver dehnbaren Lamina cribrosa begreiflich wird, ist eben so bekannt, als die anatomische Thatsache durch keine Beobachtung gestützt ist. In diesem Falle würden also jedenfalls die Excavationen nicht Druck-Excavationen sein könen, und es entsteht die Frage, ob glaucomatöse und DruckExcarationen gleichbedeutend sind, oder ob sich 
die Annahme glaucomatöser. Rand-Excavationen ohne Drucksteigerung rechtfertigen lässt.

Wenn wir, wie oben angenommen wurde, die Drucksteigerung als Folge einer gestörten Filtration aus den Aderhaut-Venen mit Ueberlastung des Glaskörperraumes (oder möglicher Weise der Papilla optica) ansehen, so wird Niemand die Möglichkeit bestreiten, dass eine geringe, lange Zeit fortwährende Hypersecretion (um ein Wort zu gebrauchen) dem Gesichtssinne sich am Aussehen der Papille offenbaren kann, ohne für den Tastsinn bemerkbar zu werden. Substituiren wir also in die Glancom-Definition anstatt einer physicalischen Anomalie des Auges seine pathologische Ursache, so liegt in einer glaucomatösen Excavation ohne Drucksteigerung kein Widerspruch.

Es fragt sich aber, welche Gründe aus der Pathologie für einen Zusammenhang der Excavation mit der vorderen Chorioidea sprechen. Mir scheinen folgende bemerkenswerth: 1. seit der Erfindung des Augenspiegels, also in 38 Jahren hat man unter den zahllosen Krankheiten des Sehnerven und der Retina nicht einen Fall gefunden, der in Excaration ausgegangen wäre; 2. vielleicht eben so ausnahmslos lässt sich der Satz für die durch den Angenspiegel bekannten Bilder der Chorio-Retinitis, Retinitis pigmentosa, Atrophien und schleichenden Entzündungen in der hinteren Hälfte des Auges aufstellen, soweit dieselben nicht von vorn übergewandert sind; 3 . je genauer man namentlich secundäre Glaucome, aber auch primäre acute und chronische, untersucht, desto zahlreicher werden die Mittheilungen und eigenen Beobachtungen von aquatorialen Plaques und Gefässdilatationen der rorderen Chorioidea; 4. die unglücklichen Ausgänge der Iridectomie wegen Glaucoma simplex lassen nie eine Beziehung zur Retina oder zum hinteren Pole der Chorioidea erkennen, sondern nur zum Corpus ciliare. Ich kenne sie in zwei Formen; entweder in der des Glaucoma malignum oder der ersten $\mathrm{Cy}$ - 
clitis acuta glaucomatoșa. Letztere sah ich neulich bei einer jugendlichen Kranken, deren rechtes Auge während eines Puerperiums unter Fieber, Bewusstlosigkeit, Ptosis, AugenMuskellähmungen, an Glancoma simplex hochgradig amblyopisch geworden war.

Dürfen wir hiernach behaupten: dass klinische Erfahrung auf einen Zusammenhang der Excavation mit der vorderen Chorioidea hinweist, - dass sich weder die Excavation des Glaucoma simplex, noch die exceptionellen Sehnervveränderungen nach Glaucoma acutum (incl. Glaucoma fulminans) mit der Annahme einer DruckExcavation vertragen, - dass nicht nur ein anatomisch genau festgestellter Raum von der Peripherie der Papilla. optica bis zur tellerförmigen Grube nicht mehr bestritten wird, sondern, dass die Einwanderung pathologischer Producte aus der Papille ebenso sicher beobachtet ist, als. die der sogenannten, cyclitischen Opacitäten, so komme ich in scheinbarem Gegensatze zur alten Glaucomlehre, in Wirklichkeit aber nur von dem Krankheitsprocesse und nicht rom Krankheits bilde ausgehend, zu folgender These: "Der in vermehrter Filtration aus dem vorderen Theile der Uvea bestehende, glaucomatöse Process ist nach einer gewissen Zeit immer an der Excavation der Papilla optica, aber keineswegs immer an fühlbarer Drucksteigerung zu erkennen."

Die Drueksteigerung bleibt das wichtigste Symptom des Krankheitshildes; denn sie gehört dem Glaucoma prodromale, subacutum, acutam and der Mehrzahl des Glaucoma simplex an und giebt dem Bilde der Chorioiditis einen dasselbe von allen anderen Entzündungen derselben Membran unterscheidenden Character. Im KrankheitsProcesse entspricht die Drucksteigerung einer vermehrten Filtration, deren minimale Grade dem 
Tastsinne entgehen, während sie an Veränderungen der Papilla optica deutlich sichtbar werden.

Durch eine Parallele der Druek-Hypothese und der von mir vertheidigten hoffe ich, die Schwächen beider übersichtlich nachweisen und diejenigen Fragen, deren Beantwortung wir, so weit möglich, dem pathologischen Anatomen überlassen müssen, genau bezeichnen zu können.

Graefe's Druek-Hypothese gebuhrt der unvergängliche Ruhm, ein neues Stück Pathologie, das umfangreiche Glaucoma secundarium und einen Theil des Glaucoma simplex - gesehaffen zu haben; an der Erklärung des Krankheits-Processes ist sie geseheitert. Wir wissen, dass Graefe ein Jahr vor seinem Tode sein Unvermőgen, Symptome und intraoculare Veränderungen in Einklang zu bringen, bekannte. Die pathologische Anatomie hatte ihn zu wenig unterstützt, seine eigene Schoppung - die Excavation - setzte ihn am meisten in Verlegenheit, da sie mit der Drucksteigerung nicht gleichen Schritt halten wollte. Die Therapie verdankt der Druek-Hypothese einen pralitischen Erfolg, dem unsere Wissenschaft im letzten Jahrhunderte keinen ebenbürtigen an die Seite stellen kann.

Graefe's Hypothese war logisch unhaltbar, deshalb auch fur die Pathologie unbrauchbar, die Prämissen waren falsch oder zweifelhaft, deshalb konnten die Folgerungen nicht richtig sein. So oft der Kliniker durch Beobachtung sein Problem zu lösen suchte, brachte sein praktisches Genie der Wissenschaft reiche Ausbeute, aber das Problem konnte auf dem eingeschlagenen Wege nicht gelöst werden. Das Wesen des glaucomatösen Processes, das gesucht wurde, konnte die Drucksteigerung nicht sein, sondern nur die Ursache der Drucksteigerung, - die Frage, ob jede Rand - Excaration eine Druck-Excavation sei, konnte ron Einem 
Kliniker in wenigen Jahren nicht entsehieden werden; denn sie ist eine rein empirische. Man darf zwar solche Entscheidungen antecipiren, um aus ihren Consequenzen auf ihre Zulässigkeit zu schliessen, aber nicht, um die Consequenzen für Wahrheiten auszugeben. War also klinisch festgestellt, dass Drucksteigerung durch Iridectomie geheilt wird, und angenommen worden, dass jede Rand-Excavation eine Druck-Excavation sei, so durfte geschlossen werden: also wird die Annahme vermuthlich richtig sein, wenn Excavationen dureh Iridectomie geheilt werden, aber keineswegs: also wird die Excavation durch Iridectomie geheilt, und für Ausnahmen von der Regel müssen besondere Gründe gesucht werden.

Bekanntlich wurde die Druck-Hypothese später vielfach mit Nichts widerlegenden und Nichts beweisenden, selten mit schlagenden, aber zu weit gehenden Gründen angegriffen. Die grosse Mehrheit der Practiker machte sich über die Druck - Excavationen bei normalem oder negativem Druck keine Sorgen, die pathologischen Anatomen konnten aus äusseren Gründen wenig helfen, hatten auch zu wenig Direction; denn Graefe konnte ihnen nicht mehr geben, als was der Augenspiegel gelehrt hatte, und positive Druck-Hypothesen, die einige Jahre hindurch wohlfeil geworden waren, erfreuten sich der Mehrzahl nach einer keineswegs ermuthigenden Begründung.

Die Leser des Archivs kann ich init der neuen Glaucom-Litteratur, in der viel Trauriges mit einigem Werthrollen vermengt ist, verschonen. Leider half uns auch, wie vorherzusehen war, die experimentelle Pathologie nicht: bei Thieren den Druck zu steigern auf die Möglichkeit hin, von den zahllosen Ursachen der Drucksteigerung die richtige, die Glaucom erzeugt, zu fassen, in thierischen Augen einen den menschlichen GlancomKrankheiten ähnlichen Process künstlich hervorzurufen, um 
für einen aus dem andern zu lernen, war ein all zu kühnes Unternehmen.

Das erste bedeutende Resultat verdanken wir der pathologischen Anatomie, der in Bd. 32 des Archivs publicirten, schönen Abhandlung von Birnbacher und Czermak. Sie beweist unwiderleglich:

1. Chorioiditis und vorzugsweise Chorioiditis anterior mit entzündlichen Verengerungen und entsprechenden Erweiterungen grosser Venen kommen bei Glaucoma acutum vor, wurden in jedem von neun untersuchten Augen nachgewiesen;

2. eine der glaucomatösen gleiche Excavation lässt sich experimentell durch Drucksteigerung herstellen;

3. die radicalen Gegner der Entzündung und Hypersecretion sind geschlagen.

Damit ist die Glaucom-Frage als eine rein empirische den pathologischen Anatomen zur Entseheidung vorgelegt. Sie haben zu untersuchen:

1. wie oft ist Chorioiditis anterior der Sectionsbefund bei Glaucoma inflammatorium;

2. wie oft finden sich bei Glaucoma inflammatorium und simplex Bedingungen für vermehrte Filtration aus den vorderen Aderhant-Venen;

3. welches ist der Befund bei Excavation mit negativem Druck?

Der Krankheitsprocess, der unter dem Bilde des acuten und einfachen Glaucom verläuft, ist also mit den Mitteln, die Graefe hinterlassen hat, unmöglich zu finden. Nur die Sections-Berichte von Birnbacher and Czermak lassen hoffen, dass der pathologische Anatom in aeut glaucomatösen Augen die Ursache der Drucksteigerung ermitteln wird. Die letzte Frage, warum es in Wirklichkeit zu 
einer Hypersecretion kommt, wenn die anatomische Möglichkeit vorbanden ist, wird vielleicht erst nach langer Zeit beantwortet werden. In Bezug auf die Therapie beisst es: wo der Druck nicht gesteigert ist, gehört die Iridectomie nicht hin.

Die von mir seit Jahren vertretene Hypothese geht ebenfalls von der Beobachtung aus, aber sie wendet sich direct vom charakteristischen Symptom zu seiner Ursache:

1. Enucleirte Augen mit Drucksteigerung bleiben härter als normale (die bisher von mir ausnahmslos gemachte Beobachtung bedarf der Bestätigung durch andere Erfahrungen). Daraus folgt

2. bei Glaucoma inflammatorium acutam, chronicum, malignum ist der Inhalt des Glaskörper-Raumes verändert resp. vermehrt. Die Richtigkeit des Schlusses ist durch eine grosse Menge klinischer Erfahrungen für alle Formen bestätigt, am wenigsten für Glaucoma simplex.

3. Anomalien des Glaskörpers sind Zeichen von Krankbeiten der Chorioidea, hydrophthalmische Producte von Dilatation und Stase in den vorderen AderhautVenen, consistente, zähe Producte von entzündlichen Vorgängen in dilatirten Venen oder von Beimischung entzündlicher Gewebsflüssigkeit zu venösem Transsudat.

In diesen Sätzen treten also an die Stelle der Drucksteigerung "die venosen Stasen im vorderen Segmente der Chorioidea" als ihre wahrscheinlichsten Ursachen. Thre Annahme beruht auf Analogien aus der Pathologie der Chorioidea und des Glaskörpers. Ueber das regelmässige Zusammentreffen muss natürlich der pathologischen Anatomie die Entscheidung bleiben. 
Eine andere, wichtige Thatsache bedarf nicht mehr der Bestätigung durch Sectionen, nämlich der anatomische Zusammenhang $z$ wischen dem Corpus ciliare und dem Central-Canal dureh den Canalis Cloquetii. Hätten wir selbst Stilling's und Flemming's anatomisehe Arbeiten nicht, so wüssten wir aus der Pathologie, dass entzundliche Producte den Weg aus der Papille zum unteren, cyclitisehe zum oberen Theile des Canales finden.

Eine Hypothese aber muss der pathologische Anatom bestätigen, wenn sich die angenommenen Glaucom-Grenzen nicht verschieben sollen: bei jedem Glaucoma simplex müssen die anatomischen Bedingungen für vermehrte Filtration aus den Venen der Aderbaut nachweisbar sein.

Lässt sich dieser Nachweis führen: so sind in der ganzen Glaucom-Reihe die Bedingungen für das Eindringen pathologischer Flüssigkeit aus der Chorioidea in die Papilla optica gegeben, und die Form - Veränderungen derselben hängen von $\mathbf{z}$ wei Factoren, von dem Eindringen der Flüsigkeit in den Centralcanal und von der intraocularen Drucksteigerung (resp. von einem von beiden) ab.

Die Druckexcavation ist durch Birnbacher und Czermak experimentell hergestellt worden, für das Eindringen eines Transsudates in die Papille sprechen das Verhalten der Function, die ophthalmoskopiseh verfolgte Entwickelung und die exceptionellen Veränderungen der Papille nach Glaucoma aeutum.

Die Hypothese, welche, conform dem altbekannten Krankheitsbilde, unter "Glaucom" alle venösen Stasen im vorderen Abschnitte der Chorioidea, deren Consequenz eine centrifugal fortschreitende Excavation der Papille ist, zusammenfasst, enthält, soweit ich sehe, keine logisehen Fehler. 
Von der pathologischen Anatomie verlangt sie: 1. den Nachweis einer in der excavirten Papille verlaufenden, vom Corpus vitreum oder dem Inhalte des Cloquet'schen Canales inducirten Krankheit; 2. den Nachweis der venösen Stasen in der vorderen Chorioidea für alle acuten und chronischen Fälle von Glaucom. (Ergäbe sich, dass bei Excavationen mit normalem oder subnormalem Drucke, die Stasen fehlen, so würden diese Fälle ausscheiden, und es wäre Sache des pathologischen Anatomen, die Ursachen der Excaration aufzufinden).

An Sectionen gerade solcher Augen, über die uns nur Leichenbefunde Aufschluss geben können, dürfte es meiner Meinung nach nicht fehlen. Wo es Siechenhäuser und grössere Stationen für Krankheiten des Greisenalters giebt, da wird man genug latente Excavationen finden und die Hauptfrage, welches der Grund der Krankheit sei, bald beantworten können.

Damit wäre denn allerdings die Glaucomlehre nicht fertig, sondern sie finge erst an, und ihre weitere Fundamentirung würde dem pathologischen Anatomen zufallen. Deshalb habe ich mich aller Träumereien uber vermehrten Zufluss und gehemmten Abfluss, über Dilatation oder Stenose des Cloquet'schen Canals enthalten, und selbst die Deutung einer Hypersecretion mit Excavation und negativem Drucke einer glücklicheren Phantasie überlassen.

Man sollte meinen, es sei für die pathologischen Anatomen der Mühe werth, zunächst das Verhalten der Chorioideal-Venen bei Glaucoma simplex zu untersuchen. Thr Verhalten bei Glaucoma inflammatorium ist, wenn auch nicht im Allgemeinen, so doch in vielen speciellen Fällen constatirt. Selbst eine Verneinung unserer Voraussetzungen wäre von Nutzen, man würde einen Irrthum aufgeben und neue Wege zu finden suchen. Mit Graefe's Lehre kann die Wissenschaft Nichts anfangen. Dem ärztlichen, therapeutischen Genie genügte sie, die Diagnose zu vervoll- 
kommnen, das Krankheitsgebiet zu erweitern, der Beobachtung neue Objecte zu bieten, das Jahrhunderte lang aufgegebene therapeutische Problem zu lösen. Was er von Bemerkungen an die dunkeln Theile seiner eigenen Lehre knüpfte, hat seinen Sehülern Anregung genug gegeben, selbstständig weiter zu forschen. Die grossen, in diesem Archive publicirten Abhandlungen werden fur alle Zeiten Beweise für das ärztliche Genie ihres Verfassers bleiben, aber von seiner Hypothese müssen wir uns lossagen, wenn wir eine Pathologie des Glaucoms schaffen wollen.

Zwischen Hypothesen über das Wesen der Krankheiten und therapeutischen Resultaten pflegt sich durch kleine Hülfs-Hypothesen leicht Harmonie herstellen zu lassen. Ich verzichte gern auf diese dankbare Operation, halte es aber für erlaubt, zu untersuchen, wie weit die der Krankenbeobachtung entnommene Hypothese ohne Zwang mit dem, was mich seit Jahren klinische Beobachtungen gelehrt haben, verträglich ist. Damit will ich schliessen.

Jeder sogenannte "Glaucom-Anfall" (auch die chronischen Glaucome verlaufen selten ganz gleichmässig) enthält zwei Factoren, die ich der Kürze wegen Disposition und Accidentelles (Gelegenheitsursache) nennen will. Ersterer ist die Hauptrolle bei dem Glaucoma chronicum, letzterer bei den acuten Formen zugetheilt: Die Bedingungen der Drucksteigerung sowohl, als der Excavation (welcher Art sie auch sein mögen), zeigen kaum eine Bewegung; in Monaten, selbst in einem Jahre lassen sich keine Verschlimmerungen der Function, Zunahme der Excavation, Steigerungen des intraoculären Druckes nachweisen, wenn nicht nach Blendung, rheumatischen Schädlichkeiten, Krankheit des Körpers, eine Steigerung eintritt, — die inflammatorischen Anfälle wiederholen sich nicht selten als prodromale, subacute nach genau derselben äusseren Veranlassung (Kartenspiel, Accommodation, Blutverlust etc.), bleiben dann lange aus und 
bis zum Rückfalle scheinen normale Verhältnisse einzutreten. Sehr viel bedeutungsvoller aber ist die Wirkung der Iridectomie; sie coupirt den acuten Anfall und heilt den Process durch Herstellung günstiger 'anatomischer Bedingungen für den Abfluss transsudirter Flüssigkeit für immer, wenn man nicht etwa annehmen will, dass sie die stationäre Disposition (senile Gefässe?) zu ändern vermag, - den Verlauf des Glancoma simplex vermag die Operation bei Weitem nicht so viel zu ändern.

Seit Graefe's Tod hat die 'Behandlung des Glaucoms Fortschritte gemacht, wir sind nicht mehr auf die Iridectomie allein angewiesen und haben die Wahl zwischen der Iridectomie, Sclerotomie oder Einträufeln von Eserin- oder Pilocarpin-Lösungen.

Die Iridectomie ist nach meinen Erfahrungen das souveräne Mittel gegen acute und chronisch-inflammatorische Formen geblieben. Die Pupille braucht weder so gross, noch grösser zu sein, wie Gräfe Anfangs verlangte, noch brauchen ihre Schenkel zu divergiren. Ihre unmittelbare Wirkung ist Verminderung der Drucksteigerung, das Auge wird sofort weicher, der Glaskörperdruck auf die Venenwand nimmt $a b$, während der vom Blutdruck $a b$ hängige intravasculäre Druck derselbe bleibt, die Blutsäule bewegt sich schneller, ihr Abfluss kann ungehindert ror sich gehen, die Stase hört allmählich auf. Wenn später unter gleichen Verhältnissen der Abfluss spontan erfolgt, müssen die anatomischen Bedingungen günstiger sein. Ich suche sie - meiner alten Annahme entsprechend - in der Unterbrechung der kreisförmigen Iris und einer Communication der allerdings lange übernarbten Colombom-Ränder mit dem Kammerwasser.

Wo ihn die Anatomie und das Experiment im Stiche lässt, darf der Kliniker auf indirecten Wegen nach Gründen suchen. Fast geheilte Iridectomie-Narben in der eigentlichen Cornea, relativ kleine Colobome schliessen die Heilung: 
des acuten Glaucoms nicht aus, aber so lange Kammerwasser fehlt, ist der Erfolg zweifelhaft. Dass das Blut der Aderhant-Venen bei Stenose der Vasa vorticosa oder bei diverticelartigen Erweiterungen des Gefässrohrs nach der Iris zurückstaut, würde uns, wenn es nicht selbstverständlich wäre, die klinische Beobachtung lehren, hier angelangt, könnte es unschädlich sein, wenn es sich in minimalen Quantitäten dem Kammerwasser beimischte und mit diesem die vorderen Lymph-Emissarien passirte. Die Ränder des Coloboms wären dann ein Sicherheits-Ventil für die Stase der Chorioiden und das um so mehr, als der Kreis-Muskel, der auch in anderen Organen die reine Hyperämie steigert, unterbrochen wäre.

Die Heilwirkung der Iridectomie gegen Glaucoma simplex mit Drucksteigerung ist mit Unrecht bestritten worden. Was ich erlebt habe, stimmt im Ganzen mit Graefe's Angaben, wenn die Zahlen anch etwas weniger günstig ausgefallen sind: geringe Besserung oder Stillstand vielleicht in der Hälfte der Fälle, in der andern erheblich verlangsamtes Fortschreiten. Die Erklärung ist: durch eine breite, periphere Iridectomie mit divergirenden Schenkeln wird zwar die Stase in den Venen der Chorioidea gemindert, aber das ununterbrochen wachsende Grundleiden (senile Gefässveränderungen?) unterhält eine Secretion, deren Intensität die neu geschaffene Abflussöffnung nicht entspricht.

Die Iridectomie s chadet: 1 . bei Glaucoma simplex mit fast den Fixirpunkt erreichendem Gesichtsfeld-Defect, das directe Sehen geht verloren, dann bleibt die Function stehen. Erklärung: Die Achsencylinder der excavirten Papille waren vom Centralcanal her nach der Richtung des maculären Bündels leitungsunfähig geworden, - mit der plötzlichen Entlastung des ganzen Auges setzt sich der pathologische Inhalt der Gruben in lebhaftere Bewegung, oder der Zufluss vom Cloquet'schen Canale her wächst, die Macula-Fasern in der Papille gehen zu Grunde; 2. bei Glaucoma malignum ohne 
Kammermasser nach der Iridectomie. Ueber das Zustandekommen habe ich eben gesprochen.

Dass die Operation wirkungslos bei normalem oder negativem Drucke ist, wird Niemand befremden. In allem Uebrigen scheint mir- die Hypothese mit der Therapie ohne Zwang vereinbar, wenn wir die unmittelbare Wirkung der Iridectomie von der späteren, palliativen und die Prädisposition von der directen Ursache des Anfalles trennen.

Wollte man die ungenügende Wirkung der Sclerotomie zu Gunsten meiner Hypothese ausbeuten, so läge der Weg klar genug da: der Kreismuskel, die Iris, bleibt unversehrt, die Berïhrung der Wundränder mit dem Kammerwasser ist ausgeschlossen, aber wir wissen nicht, was in dem bald blutenden, bald blutleeren Stichkanale vor sich geht and speisen uns mit der Filtrationsnarbe ab, können also über die Art der Entlastung für die Chorioidea nicht urtheilen. Als Ersatz für die Iridectomie; wo eine gar zu flache Kammer die Ausführung erschwerte und als Refugium, wenn ich Glaucoma malignum fürchtete, war sie mir sehr willkommen. Der Iridectomie ist weder ihre augenblickliche, noch palliative Wirkung ebenbürtig.

Dass das mächtig gefässverengende Eserin durch Beschleunigung des Blutstromes in der Iris den acuten Anfällen gewachsen sein, aber nur äusserst selten Recidive verhüten kann, spricht für die Nothwendigkeit des künstlichen Iris-Coloboms, dass das schwächer in gleichem Sinne wirkende Pilocarpin im acuten Anfalle Nichts leistet, bei Monate und Jahre langem Gebrauche aber sich immer mehr gegen Glaucoma simplex bewährt, harmonirt vortrefflich mit der unmerklich wachsenden Druck-Ursache, deren Folgen durch täglich mehrmals herbeigeführte Beschleunigung des Abflusses aus der Iris annullirt werden. Bei beiden Myoticis dürfte noch zu beachten sein, was unter der Alleinherrschaft der lymph-Retention kaum der Rede werth schien, dass Myotica die Tris verlăngern, Atropin dieselbe verkürzt, 
seine gefässlähmende Kraft die Stase in einer verbürzten Fläche, also sehr wohl hervorrufen und Glaucom erzeugen kann, wenn auch der schöne Traum von den Fontana'schen Räumen sich nicht, verwirklichen sollte.

Veranlassung, von Neuem auf "das Wesen des Glancoms zurückzukommen, gab mir vor Allem die oft citirte Abhandlung von Birnbacher und Czermak mit ihren positiven Resultaten, gegen deren Sicherheit man schwerlich etwas einwenden wird.

Gegen die Lymph-Hypothese, deren räthselhaften Erfolg ich mir aus Allem eher erklären konnte, als aus ihrer Begrundung, habe ich von ihrer ersten Veröffentlichung bis zum heutigen Tage einzuwenden gehabt, dass sie in der ganzen Pathologie als ein die Krankheitserscheinungen schlecht erklärendes Unicum dasteht.

Als mein alter Freund Hippel vor etwa 15 Jahren seine schönen Versuche mit Grünhagen, um Glaucom zu erzeugen, begann, äusserte ich schon meine Bedenken dahin, er werde den intraocularen Druck steigern, aber nicht Glaucom erzeugen; denn letzteres scheine mir gewisse, im menschlichen Auge meist im Alter entstehende Gewebsveränderungen vorauszusetzen. Die Wissenschaft kann zufrieden sein, dass die Versuche nicht unterblieben sind, meinen Befürehtungen hat der Erfolg leider Recht gegeben.

Wie viel Jahre lang man nachher noch Druck gesteigert hat und mit welchem Resultate für die Glaucomlehre, ist den Lesern des Archivs bekannt. Graefe selbst war die unschuldige Ursache, dass man ein Symptom für das Wesen hielt und die Krankheit erzeugen zu können vermeinte, wenn man ein Symptom experimentell zu Stande brachte. 
Die späteren Anhänger der Lymph-Retention haben es principiell richtiger angefangen,"indem sie eine directe, mechanische Ursache des constanten Symptoms, der Drucksteigerung, suchten, und diese mit den klinisch bekannten Umständen, unter denen Glaucom zu entstehen pflegt, so gut es ging, in causalen Zusammenhang brachten. Meiner Meinung nach haben sie dabei weniger ärztliche, als. augenärztliche Befähigung gezeigt.

Die Exclusion der Chorioiditis, wie sie de Wecker noch in seinem neuen Lehrbuche vertritt, ist misslungen. Was Birnbacher und Czermak in der Chorioidea und ihren Gefässen gefunden haben, lässt sich nicht ignoriren, zumal da es durch eine nicht geringe Zahl von Präparaten anderer Autoren bestätigt wird. Für den pathologischen Anatomen wird es immer eine schwierige Aufgabe sein, seine Entscheidung über die Abhängigkeit des Glaucoms von einer Lymph-Retention abzugeben, selbst wenn er eine Menge Emissarien verschlossen findet.

Die Frage, ob bei allen centrifugal fortschreitenden oder, um bei Graefe zu bleiben, bei allen Rand-Excavationen sich im vorderen Abschnitte der Chorioidea oder in den Vasa vorticosa Bedingungen für vermehrte Transsudation nachweisen lassen, scheint mir deshalb die nächste zu sein, die beantwortet werden muss. Von ihrer Entscheidung hängt es ab, ob der Kliniker auf dem angegebenen Wege fortfahren darf, oder neue Richtungen zu suchen haben wird.

Es ist nicht zum ersten Male, dass ich an die unzweckmässige Disposition unserer Untersuchungen und Discussionen tiber die Glancom-Frage erinnere, aber zum ersten Male erfreue ich mich der Zustimmung zweier Collegen, deren erstes Auftreten ihrem Urtheil ein nicht geringes Gewicht sichert. Die Ophthalmopathologie wird noch für lange Zukunft von dem Kliniker mehr fordern, als 
andere, klinische Disciplinen, um so mehr, je weniger der pathologische Anatom im Stande ist, Fragen, die in sein Gebiet allein gehören, zu beantworten.

Verdanken wir es nun auch Helmholtz, uns über viele pathologisehe Vorgänge in der binteren Hälfte des Auges ohne Sectionen einigermassen orientiren za können, so haben wir doch immer noch $z u$ beklagen, dass etwa von der Gegend des Linsenrandes bis zum Aequator des Auges weder objective, noch subjective Symptome uns einen Einblick in die Pathologie der Chorioidea gestatten. Wir sind auf Conjecturen aus der Beschaffenheit des Corpus vitreum, des Humor aqueus, der Consistenz des Auges etc. angewiesen und haben von Sectionen, die vielleicht 10-20 Jahre nach Ablauf der Krankheit vorgenommen werden, wenig zu erwarten.

Um so dringender ist es geboten, in den seltenen Fällen, in denen der Kliniker indirect den Sitz einer Krankheit gefunden zu haben glaubt, und Gelegenheit genug zu Sectionen sich bietet, die Entscheidung des pathologisehen Anatomen herbeizuführen. Für die Frage, ob venöse Stasen in der vorderen Chorioidea zum regelmässigen Sectionsbefunde des Glaucoma simplex gehören, lässt sich ein nicht geringes Material ohne grosse Schwierigkeit auftreiben.

$\mathrm{Ob}$ die Annahme sich bestätigt, ist für die weitere klinische Beobachtung allein massgebend; denu jede sichtbare pathologische Veränderung im Innern des Auges erhält ihren Sinn von dem Grundleiden, als dessen Consequenz sie sich entwickelt; geringe Abweichungen von der Norm, die der Beachtung nicht werth scheinen, werden zu wichtigen Symptomen durch ihre Beziehungen zu einem sicher erkannten Krankheits-Processe.

Entzündliche Vorgänge und Anomalien der Venen, die im Glaucoma inflammatorium vorkommen können, sind unwiderleglich nachgewiesen. Es handelt sich darum, ob ähnliche mechanische Hindernisse des Blutstroms auch bei Exv. Graefe's Archiv für Ophthalmologie, XXXIV. 1. 
cavationen der Papille die Regel sind. Dann wird man wissen, ob man alle Excavationen für Folgen venöser Stauung, für Producte des vorläufig hypothetischen, glaucomatősen Processes halten darf und den causalen Zusammenhang zu begreifen suchen. Die Definition und die klinische Grenze des Glancoms aber wäre schon durch die Bestätigung gegeben. 